\title{
Exenteração Pélvica: Relato de Caso
}

\author{
Pelvic Exenteration: a case Study \\ Exenteración Pélvica: Reporte de un Caso
}

Alexandra Isabel de Amorim Lino', Cristine Alves Costa de Jesus²

\begin{abstract}
RESUMO
Trata-se de um relato de caso de um paciente com colostomia úmida, após exenteração pélvica total, atendido em uma instituição hospitalar pública de saúde, localizada na cidade de Brasília (DF), Brasil. O objetivo geral foi a aplicação do processo de enfermagem em indivíduo no pós operatório mediato de exenteração pélvica total com colostomia úmida. A coleta de dados foi realizada no mês de setembro de 2013, por meio de entrevista e exame físico com duração de aproximadamente 40 minutos. Utilizou-se um instrumento elaborado pelas autoras, o qual foi fundamentado no referencial teórico de Wanda Horta, das Necessidades Humanas Básicas. Foram levantados os diagnósticos de enfermagem, resultados e intervenções pertinentes para este caso. Os diagnósticos identificados abordam, essencialmente, as necessidades psicobiológicas. Dentre os diagnósticos que subsidiaram o atendimento a esse paciente, temos: a Integridade tissular prejudicada, distúrbio na imagem corporal, incontinência intestinal, conhecimento deficiente, dor aguda, déficit para o autocuidado para banho, padrão de sono prejudicado, mobilidade física prejudicada e risco de infecção. Este relato confirma que uma assistência de enfermagem sistematizada promove um cuidado direcionado e um registro preciso e embasado cientificamente, que possibilita a continuidade da assistência de qualidade em conjunto com o cliente.
\end{abstract}

DESCRITORES: Enfermagem. Diagnóstico de Enfermagem. Colostomia.

\begin{abstract}
This is a case study of a patient with wet colostomy, after having been undergone to a total pelvic exenteration treatment, attended in a public hospital institution located in Brasília/DF. This investigation aimed to apply the nursing procedure in an individual who was in the immediate postoperative phase due to a total pelvic exenteration treatment. This person had wet colostomy. The data collection was carried out in September, 2013, through interviews and physical examination and it lasted about 40 minutes. We used an instrument developed by the authors, which was based on the theoretical framework of Wanda Horta on the Basic Human Needs. The nursing diagnoses, its results and relevant interventions to this case were surveyed. The identified diagnoses essentially address the psychobiological needs. Among the diagnostics that supported the aid to the referred patient, we have the following: tissue integrity, disturbance in body image, bowel incontinence, deficiency of knowledge, acute pain, deficits self-caring when taking baths, disturbed sleep pattern, impaired physical mobility and risk of infection. This case study confirms that a systematic nursing care promotes a guided care and an accurate scientifically grounded record that enables the continuity of quality care, along with the customer.
\end{abstract}

DESCRIPTORS: Nursing. Nursing Diagnosis. Colostomy. 


\section{RESUMEN}

Se trata de un caso clínico de un paciente con colostomía húmeda, después de la exenteración pélvica total, atendido en un hospital público, que se encuentra en Brasilia (DF), Brasil. El objetivo fue la aplicación del proceso de enfermería en el individuo en la exenteración pélvica inmediata postoperatoria total con colostomía húmeda. La recolección de datos se llevó a cabo en septiembre de 2013, a través de entrevistas y el examen físico que duran unos 40 minutos. Se utilizó un instrumento desarrollado por los autores, que se basan en el marco teórico de Wanda Horta, sobre las Necesidades humanas básicas. Diagnósticos de enfermería, resultados e intervenciones pertinentes a este caso se plantearon. Los diagnósticos identificados abordan esencialmente las necesidades psicobiológicas, a partir de los diagnósticos que apoyaron el cuidado de este paciente, tenemos: la integridad del tejido dañado, alteración de la imagen corporal en, incontinencia intestinal, la falta de conocimiento, dolor agudo, los déficit en autocuidado en el baño, trastornos del sueño, alteración de la movilidad física y el riesgo de infección. Este informe confirma que un cuidado de enfermería sistemática promueve una atención dirigida y un registro exacto y científicamente a tierra que permite la continuidad de la atención de calidad junto con el cliente.

PALABRAS CLAVE: Enfermería. Diagnóstico de enfermería. Colostomía.

\section{INTRODUÇÃO}

Para se estabelecer um cuidado de qualidade se faz necessário utilizar o processo de enfermagem, que promove a organização e representa um método pelo qual o enfermeiro realiza a assistência sistematizada e direcionada a esse cuidado. $\mathrm{O}$ enfermeiro que assiste o indivíduo de forma sistematizada promove um cuidado integral e individualizado, a fim de atender as especificidades na situação clínica determinada ${ }^{1}$.

Promover um cuidado diferenciado exige o uso de um padrão na linguagem, para uma comunicação eficiente na identificação dos sinais e sintomas reais ou potenciais apresentados pelo cliente, dando mais responsabilidade e reconhecimento ao enfermeiro ${ }^{1,2}$.

A satisfação do indivíduo envolve uma variedade de fatores, particularmente o cliente com câncer de reto diagnosticado irá depender do conhecimento do enfermeiro e demais profissionais da equipe para proporcionar um entendimento de todas as intervenções por parte do individuo, uma vez que o tratamento propõe uma alteração fisiológica do trato gastrointestinal que interfere psicologicamente na qualidade de vida e readaptação do indivíduo.

Estudos apontam que cerca de 6 a 10\% das neoplasias retais são diagnosticadas em estádios avançados (T4) e, após o procedimento cirúrgico curativo, tem recidiva de 4 a 50\% dos casos, impactando na qualidade de vida e sobrevida do cliente. Com o intuito de melhorar a qualidade de vida e sobrevida, tem-se realizado a exenteração pélvica, inicialmente idealizada e utilizada em clientes com neoplasia de colo de útero recidivado, descrita por Brunschwig em 1948.
Os órgãos envolvidos na exenteração que podem ser infiltrados são: ureteres, bexiga, próstata, vesículas seminais, ovários, útero, vagina, alças intestinais e parede pélvica ${ }^{3,4}$.

No entanto, representa uma morbimortalidade alta, mas é a única alternativa curativa ao câncer de reto localmente avançado. Porém, nota-se descrito na literatura uma diminuição das taxas de morbimortalidade em decorrência do avanço da técnica cirúrgica e do suporte perioperatório, devido à indicação adequada. A sobrevida do cliente submetido ao procedimento cirúrgico é maior (de 60\% em 5 anos) quando comparado a condições em que se utilizam apenas o tratamento com quimioterapia e radioterapia (cerca de 5\% em 5 anos) $)^{3,4}$.

O sucesso do tratamento cirúrgico envolve não apenas o componente relativo à equipe médica, mas também uma assistência de enfermagem qualificada e sistematizada. O processo de enfermagem dá suporte para a qualificação exigida para a garantia de um cuidado efetivo e diferenciado.

Os objetivos do estudo foram: aplicar o processo de enfermagem em indivíduo pós exenteração pélvica com colostomia úmida, apoiado em sistemas de classificação em enfermagem; e Promover a assistência de enfermagem a partir dos diagnósticos identificados da Taxonomia da NANDA-I e do uso das Classificações de Intervenções (NIC) e Resultados (NOC).

\section{MÉTODO}

Trata-se de relato de caso em que se buscou aplicar o processo de enfermagem em indivíduo pós exenteração 
e promover a assistência de enfermagem a partir dos diagnósticos identificados da Taxonomia da NANDA-I e do uso das Classificações de Intervenções (NIC) e Resultados (NOC).

A amostra foi constituída por um paciente atendido em uma instituição hospitalar pública de saúde, localizada na cidade de Brasília (DF), que aceitou participar da pesquisa e assinou o Termo de Consentimento Livre e Esclarecido. O estudo foi autorizado pelo Comitê de Ética em Pesquisa da Fundação de Ensino e Pesquisa da Escola de Ciências da Saúde (FEPECS) sob o número de parecer 313.309. Foram cumpridas as exigências da Resolução no 466/2012, do Conselho Nacional de Saúde/ Ministério da Saúde. A coleta de dados foi realizada no mês de setembro de 2013, por meio de entrevista e exame físico, com duração de aproximadamente 40 minutos. $O$ paciente foi abordado na enfermaria do hospital. Para a coleta de dados utilizou-se um instrumento elaborado pelas autoras, o qual foi fundamentado no referencial teórico de Wanda Horta, das Necessidades Humanas Básicas. O referido instrumento sofreu validação por dois enfermeiros com experiência em diagnósticos e atendimento de clientes em condições cirúrgicas.

O instrumento de coleta de dados possibilitou o registro dos dados da entrevista e do exame físico e a aplicação do processo de enfermagem subsidiado nas Taxonomias de diagnósticos, intervenções e resultados, NANDA-I, NIC e NOC, respectivamente. Outros dados foram complementados do prontuário eletrônico do paciente.

\section{RESULTADOS}

Será apresentada, a seguir, uma síntese do caso estudado, no qual estarão em destaque os aspectos que direcionaram para a elaboração dos diagnósticos de enfermagem.

\section{Caso clínico}

EBS, de 39 anos, sexo masculino, aposentado pelo INSS, HIV positivo em uso de antirretrovirais, com diagnóstico médico de neoplasia de reto, ânus e canal anal. Inicialmente submetido à sigmoidectomia devido a múltiplas fístulas em região perineal, após tratamento prévio com quimioterapia e radioterapia. Após 3 meses com melhora do quadro infeccioso, foi submetido a exenteração pélvica total, na tentativa de cura, tendo ocasionado uma colostomia úmida terminal, com saída de urina e fezes pelo mesmo estoma. Encontrava-se consciente, orientado, calmo, comunicativo. Necessidades psicobiológicas: apresentava-se eupneico, tórax simétrico com murmúrios vesiculares presentes em toda a extensão, sem presença de ruídos adventícios, com tosse seca esporádica, a pressão arterial aferida foi de $133 \times 80 \mathrm{mmHg}$, pulso de $62 \mathrm{bpm}$, perfusão capilar normal com rede venosa visível e sem alterações. Hidratação e Nutrição: Apresentava abdome plano flácido, ruídos hidroaéreos presentes em todos os quadrantes, colostomia úmida à esquerda, com aceitação parcial da dieta oral. Eliminação: apresentava saída de urina por colostomia úmida, sem presença ainda de fezes no segundo dia de pós operatório. Sono e Repouso: sono alterado mesmo com uso de medicação para dormir. Locomoção: com alterações da locomoção devido à necessidade de repouso por ressecção extensa acometendo nádegas. Integridade cutâneo-mucosa: apresentava pele hipocorada, sem alterações de temperatura ou no local do sitio cirúrgico. Sem alterações de estoma e ou pele periestomal. Necessidades Psicossociais (segurança, conhecimento e comunicação): tinha conhecimento da internação, mas não conhece sobre toda a doença e tratamento. É alfabetizado, apresenta comunicação verbal normal, na comunicação não verbal expressava dor, apresentava dificuldade de aprendizado por ainda estar em processo de aceitação. A família conhecia, mas apenas o companheiro e a amiga participavam do processo de tratamento e recuperação. Apresentava solidão, embora tivesse motivação para tratamento e capacidade para o autocuidado. Ainda tinha dificuldades na aceitação da mudança na autoimagem. Necessidades Psicoespirituais: referiu ser católico praticante.

O Quadro 1 traz os diagnósticos de enfermagem da NANDA-I estabelecidos, bem como os resultados da NOC e intervenções de enfermagem da NIC.

\section{DISCUSSÃO}

Neste caso, identificamos que a maioria dos diagnósticos de enfermagem priorizados estava na categoria de necessidades psicobiológicas.

O referencial teórico de Wanda Horta, apoiado na hierarquia das necessidades humanas (necessidades 
Quadro 1. Síntese a Sistematização da Assistência de Enfermagem pela NANDA, Classificações de Intervenções e Resultados.

\begin{tabular}{|c|c|c|}
\hline Diagnósticos de Enfermagem & NOC & NIC \\
\hline $\begin{array}{l}\text { Integridade tissular prejudicada } \\
\text { relacionada a fatores mecânicos } \\
\text { evidenciado por tecido lesado. }\end{array}$ & $\begin{array}{l}\text { INTEGRIDADE TISSULAR } \\
\text { - PELE E MUCOSAS: } \\
\text { Aumentar perfusão } \\
\text { tissular (3) para levemente } \\
\text { comprometimento } \\
\text { (4) / Aumentar para } \\
\text { integridade da pele (5) não } \\
\text { comprometida (5). }\end{array}$ & $\begin{array}{l}\text { CUIDADOS COM LOCAL DE INCISÃO: } \\
\text { Monitorar o processo de cicatrização no } \\
\text { local da incisão; orientar o paciente sobre } \\
\text { as formas de cuidar da incisão durante } \\
\text { o banho; ensinar ao paciente como } \\
\text { minimizar a pressão sobre o local da } \\
\text { incisão. SUPERVISÃO DA PELE: Monitorar } \\
\text { a pele quanto ao ressecamento e } \\
\text { umidade excessivos; examinar a } \\
\text { condição da incisão cirúrgica, conforme } \\
\text { apropriado. }\end{array}$ \\
\hline $\begin{array}{l}\text { Distúrbio na imagem corporal } \\
\text { relacionado à doença e } \\
\text { à cirurgia evidenciados } \\
\text { por resposta não verbal a } \\
\text { mudanças reais no corpo. }\end{array}$ & $\begin{array}{l}\text { IMAGEM CORPORAL: } \\
\text { Aumentar adaptação a } \\
\text { mudanças na aparência } \\
\text { física e nas funções do } \\
\text { corpo para frequentemente } \\
\text { positivo (4). }\end{array}$ & $\begin{array}{l}\text { MELHORA DA IMAGEM CORPORAL: } \\
\text { Ajudar o paciente a discutir mudanças } \\
\text { causadas por doença ou cirurgia, } \\
\text { conforme apropriado; monitorar se o } \\
\text { paciente consegue olhar para a parte do } \\
\text { corpo modificada; identificar grupos de } \\
\text { apoio disponíveis ao paciente. }\end{array}$ \\
\hline $\begin{array}{l}\text { Incontinência intestinal e } \\
\text { urinária relacionadas às lesões } \\
\text { colorretais; perda do controle } \\
\text { do esfíncter anal, evidenciado } \\
\text { por incapacidade de reconhecer } \\
\text { a pressão para evacuar, para } \\
\text { retardar evacuação e perda } \\
\text { constante de fezes amolecidas. }\end{array}$ & $\begin{array}{l}\text { ELIMINAÇÃO INTESTINAL: } \\
\text { Aumentar padrão de } \\
\text { eliminação para levemente } \\
\text { comprometido (4). }\end{array}$ & $\begin{array}{l}\text { CUIDADOS COM ESTOMIAS: Aplicar o } \\
\text { dispositivo de estomia de forma que } \\
\text { se adapte de forma adequada, sempre } \\
\text { que necessário; trocar e esvaziar a bolsa } \\
\text { de ostomia sempre que apropriado; } \\
\text { monitorar padrões de eliminação. }\end{array}$ \\
\hline $\begin{array}{l}\text { Conhecimento deficiente } \\
\text { relacionado à falta de } \\
\text { exposição, falta de familiaridade } \\
\text { com os recursos de informação, } \\
\text { evidenciado por verbalização do } \\
\text { problema. }\end{array}$ & $\begin{array}{l}\text { CONHECIMENTO - } \\
\text { CUIDADO DA OSTOMIA: } \\
\text { Propósito da estomia } \\
\text { (1) aumentar para } \\
\text { conhecimento substancial } \\
\text { (4) / Suprimentos } \\
\text { necessários para } \\
\text { cuidar de uma estomia } \\
\text { (1) aumentar para } \\
\text { conhecimento substancial } \\
\text { (4) CONHECIMENTO - } \\
\text { CUIDADOS NA DOENÇA: } \\
\text { Regime de tratamento } \\
\text { (3) aumentar para } \\
\text { conhecimento substancial } \\
\text { (4) / Regime de } \\
\text { tratamento (3) aumentar } \\
\text { para conhecimento } \\
\text { substancial (4). }\end{array}$ & $\begin{array}{l}\text { CUIDADOS COM ESTOMIAS: Orientar o } \\
\text { paciente / pessoa importante quanto } \\
\text { ao uso de dispositivo / cuidados com } \\
\text { ostomias; fazer o paciente / pessoa } \\
\text { importante demonstrar o uso do } \\
\text { equipamento; monitorar a cicatrização } \\
\text { da incisão/ estoma; explicar ao paciente } \\
\text { quais mudanças o cuidado da ostomia } \\
\text { trará para sua rotina diária; encorajar } \\
\text { a participação em grupos de apoio a } \\
\text { estomizados após a alta. ENSINO - } \\
\text { PROCESSO DA DOENÇA: Avaliar o nível } \\
\text { atual de conhecimento do paciente } \\
\text { relativo ao determinado processo } \\
\text { de doença; descrever os sinais e os } \\
\text { sintomas comuns da doença, conforme } \\
\text { apropriado; dar informaçoses ao } \\
\text { paciente sobre a condição, conforme } \\
\text { apropriado; discutir opções de terapia } \\
\text { / tratamento; orientar o paciente sobre } \\
\text { medidas de controle / minimização } \\
\text { de sintomas, conforme apropriado; } \\
\text { reforçar as informações dadas por } \\
\text { outros membros da equipe de saúde, } \\
\text { conforme apropriado. }\end{array}$ \\
\hline
\end{tabular}


Quadro 1. Continuação.

\begin{tabular}{|c|c|c|}
\hline Diagnósticos de Enfermagem & NOC & NIC \\
\hline $\begin{array}{l}\text { Dor aguda relacionada a agentes } \\
\text { lesivos evidenciada por relato } \\
\text { verbal de dor e expressão facial. }\end{array}$ & $\begin{array}{l}\text { CONTROLE DA DOR: } \\
\text { Diminuir o relato de } \\
\text { dor (5) para raramente } \\
\text { demonstrado (2). }\end{array}$ & $\begin{array}{l}\text { CONTROLE DA DOR: Observar a ocorrência } \\
\text { de indicadores não verbais de desconforto, } \\
\text { em especial nos pacientes incapazes de se } \\
\text { comunicar com eficiência; assegurar que } \\
\text { o paciente receba cuidados precisos de } \\
\text { analgesia; investigar junto ao paciente os } \\
\text { fatores que aliviam / pioram a dor. }\end{array}$ \\
\hline $\begin{array}{l}\text { Défict de autocuidado para } \\
\text { banho relacionado à fraqueza } \\
\text { e à cirurgia, evidenciado por } \\
\text { incapacidade de regular a } \\
\text { água do banho e de acessar o } \\
\text { banheiro. }\end{array}$ & $\begin{array}{l}\text { AUTOCUIDADO - BANHO: } \\
\text { Aumentar a obtenção } \\
\text { dos itens para o banho, } \\
\text { obtenção de água para } \\
\text { o banho e possibilidade } \\
\text { de banhar-se com o } \\
\text { chuveiro para levemente } \\
\text { comprometido (4). }\end{array}$ & $\begin{array}{l}\text { ASSISTÊNCIA NO AUTOCUIDADO: } \\
\text { Monitorar a capacidade do paciente para } \\
\text { autocuidado independente; oferecer os } \\
\text { artigos pessoais desejados. ASSISTÊNCIA } \\
\text { NO AUTOCUIDADO - BANHO/HIGIENE: } \\
\text { Colocar toalhas, sabonete, desodorante, } \\
\text { material de barba e outros acessórios no } \\
\text { banheiro; providenciar os itens pessoais } \\
\text { desejados; facilitar ao paciente a escovação } \\
\text { dos dentes, conforme apropriado; facilitar } \\
\text { que o paciente tome banho sozinho, } \\
\text { conforme apropriado; oferecer assistência } \\
\text { até que o paciente fique totalmente capaz } \\
\text { de assumir o autocuidado. }\end{array}$ \\
\hline $\begin{array}{l}\text { Padrão de sono prejudicado } \\
\text { relacionado à falta de } \\
\text { privacidade/controle do sono, } \\
\text { iluminação, ruído evidenciado } \\
\text { por relatos de dificuldade para } \\
\text { dormir e mudança no padrão } \\
\text { normal de sono. }\end{array}$ & $\begin{array}{l}\text { SONO: Diminuir o sono } \\
\text { interrompido (2) } \\
\text { para levemente } \\
\text { comprometido (4). }\end{array}$ & $\begin{array}{l}\text { MELHORA DO SONO: Adaptar o ambiente; } \\
\text { determinar o padrão de sono / vigília } \\
\text { do paciente; ajustar os horários de } \\
\text { administração de medicamentos em } \\
\text { apoio ao ciclo de sono; monitorar / } \\
\text { registrar o padrão de sono e o número de } \\
\text { horas de sono do paciente. }\end{array}$ \\
\hline $\begin{array}{l}\text { Mobilidade física prejudicada } \\
\text { relacionada à dor e ao } \\
\text { desconforto, evidenciado } \\
\text { por capacidade diminuída de } \\
\text { desempenhar as habilidades } \\
\text { motoras finas e grossas. }\end{array}$ & $\begin{array}{l}\text { MOBILIDADE: Aumentar } \\
\text { equilíbrio, coordenação e } \\
\text { marcha para levemente } \\
\text { comprometido (4). }\end{array}$ & $\begin{array}{l}\text { TERAPIA COM EXERCÍCIO - DE } \\
\text { AMBULAÇÃO: Colocar o controle da cama } \\
\text { ao alcance da mão do paciente; encorajar } \\
\text { a sentar na cama, na lateral da cama ou } \\
\text { em poltrona, conforme tolerância; auxiliar } \\
\text { o paciente a transferir-se, se necessário; } \\
\text { ajudar o paciente na deambulação inicial e } \\
\text { conforme necessidade; orientar paciente } \\
\text { sobre técnicas seguras de transferência e } \\
\text { deambulação. TERAPIA COM EXERCícIO - } \\
\text { EQUILÍBRIO: Determinar a capacidade do } \\
\text { paciente para participar de atividades que } \\
\text { exijam equilíbrio; orientar o paciente sobre } \\
\text { a importância da terapia com exercícios } \\
\text { para manter e melhorar o equilíbrio; } \\
\text { oferecer dispositivos auxiliares para apoio } \\
\text { do paciente ao realizar os exercícios. }\end{array}$ \\
\hline $\begin{array}{l}\text { Risco de infecção relacionado a } \\
\text { defesas primárias inadequadas. }\end{array}$ & $\begin{array}{l}\text { CONHECIMENTO- } \\
\text { CONTROLE DE INFECÇÃO: } \\
\text { Manter em conhecimento } \\
\text { substancial (4) os sinais } \\
\text { e sintomas de infecção / } \\
\text { Aumentar para conhecimento } \\
\text { substancial (4) a importância } \\
\text { da higienização das mãos. }\end{array}$ & $\begin{array}{l}\text { PROTEÇÃO CONTRA INFECÇÃO: } \\
\text { Monitorar sinais, sintomas e locais de } \\
\text { infecção; examinar a pele e as mucosas } \\
\text { em busca de hiperemia, calor extremo } \\
\text { ou drenagem; examinar as condições } \\
\text { de todas as incisões/feridas cirúrgicas; } \\
\text { promover ingestão nutricional adequada; } \\
\text { estimular a ingestão hídrica. }\end{array}$ \\
\hline
\end{tabular}


psicobiológicas, necessidades psicossociais e necessidades psicoespirituais), demonstrou ser aplicável à situação e foi importante para o direcionamento dos dados, bem como priorização dos diagnósticos de enfermagem. Embora o referencial de Horta não aborde os diagnósticos de enfermagem, como atualmente tem sido utilizado, direcionado a um sistema atual de classificação (NANDA-I), não houve conflito entre eles. Cada uma das necessidades humanas foi contemplada com classes que indicavam as especificidades da classificação (oxigenação, segurança, hidratação, nutrição etc. $)^{5}$.

A adoção de um referencial teórico de enfermagem garante uma explanação sistemática de eventos para descrever, explicar e entender os fenômenos e a solucionar os problemas, auxiliando o entendimento do enfermeiro acerca da prática e do conhecimento existente ${ }^{6,7}$.

A aceitação e entendimento da situação tem um desenrolar diferenciados quando há uma organização nesse processo, pois o cliente se sente amparado e tende a uma readaptação menos traumática.

Quanto à integridade tissular prejudicada, podemos ter um acometimento da mucosa intestinal e /ou da pele periestoma, o que desejamos é proporcionar uma boa aderência do equipamento coletor adaptado para preservar a pele periestomal, no caso em questão o paciente apresentou uma boa aderência à base adesiva, com uso da pasta para nivelar pele periestomal e bolsa opaca. $\mathrm{O}$ uso de adjuvantes permite a manutenção da pele periestoma íntegra ${ }^{10,11}$

A falta de controle voluntário dos efluentes pela estomia é um fator limitante, porém a adequação do tipo de equipamento coletor com protetor de pele, filtro de carvão ativado e adjuvantes podem favorecer a segurança e bem-estar do estomizado, minimizando as consequências negativas do procedimento cirúrgico. $\mathrm{O}$ retorno social do indivíduo, uma vez que o odor, os ruídos e o volume do efluente dificultam o convívio fora do domicílio, nomeado, geralmente, como um local seguro para o estomizado ${ }^{12}$, obstáculos que favorecem o distúrbio na imagem corporal e podem estimular pensamentos negativos, originando a depressão em decorrência da alteração física imposta ${ }^{2}$.

O déficit de conhecimento se atribui à falta de orientação e insuficiência de informação específica. Em outro estudo desenvolvido ${ }^{13}$, também foi predominante entre os estomizados. Para tanto, o ensino do paciente de forma precoce diminui a dúvida e a incerteza sobre os procedimentos realizados. Neste caso, o paciente foi acompanhado no ambulatório de estomaterapia desde o primeiro procedimento cirúrgico, que originou em uma estomia intestinal, o que resultou em um melhor entendimento e adaptação à estomia.

A dor aguda, ainda muito presente dentre as queixas mais comuns referidas pelos pacientes em pós-operatório, tem sido identificada como um problema de saúde pública que necessita ser sanado ${ }^{14}$. Quando associada ao câncer, pode ter origem física, emocional e social, e, nesse contexto, é esperado que 70 a $80 \%$ dos pacientes com doença oncológica experimentem uma dor de moderada a intensa ${ }^{15}$, além de apresentar alteração do padrão de sono.

O padrão de sono prejudicado decorre do ambiente hospitalar, uma vez que sua alteração se deve a interrupções da qualidade e quantidade de sono por fatores externos ${ }^{2}$.

O risco de infecção está presente pela exposição a patógenos e procedimentos invasivos ${ }^{2}$, portanto todo o paciente internado se não apresentar infecção apresenta esse diagnóstico.

\section{CONSIDERAÇÕES FINAIS}

Os diagnósticos identificados abordam, essencialmente, as necessidades psicobiológicas, dentre os diagnósticos que subsidiaram o atendimento a esse paciente, temos: a integridade tissular prejudicada, distúrbio na imagem corporal, incontinência intestinal, conhecimento deficiente, dor aguda, déficit para o autocuidado para banho, padrão de sono prejudicado, mobilidade física prejudicada e risco de infecção.

Este relato confirma que uma assistência de enfermagem sistematizada promove um cuidado direcionado e um registro preciso e embasado cientificamente, que possibilita a continuidade da assistência de qualidade em conjunto com o cliente.

Assim, se reafirma a necessidade do conhecimento teórico prático do enfermeiro no período perioperatório, proporcionando um acolhimento e uma satisfação do paciente em relação aos procedimentos realizados.

Obteve-se, como confirmação, que o ensino do paciente deve ser gradual e individual, desde o momento do pré-operatório mediato. Quanto à avaliação do plano 
de cuidados estipulados para este caso, apresentou-se resolução parcial apenas para os diagnósticos de distúrbio da imagem corporal e a incontinência intestinal, que, apesar de o paciente aceitar a colostomia úmida, necessita de estímulos positivos diariamente para se manter sem pensamentos negativos.
O paciente recebeu acompanhamento, mesmo após a alta na unidade, em nível ambulatorial, o que permitiu maior entrosamento e continuidade do cuidado.

O tema tem pouco destaque na enfermagem brasileira e mais estudos devem ser realizados para que se possa oferecer um cuidado diferenciado a essa clientela.

\section{REFERÊNCIAS}

1. Tannure MC, Gonçalves AMP. Sistematização da Assistência de Enfermagem, Rio de Janeiro: Guanabara Koogan; 2008.

2. NANDA Internacional. Diagnósticos de Enfermagem da NANDA: Definições e classificação. Porto Alegre: Artmed; 2012.

3. Costa SRP, Teixeira ACP, Lupinacci RA. A exenteração pélvica para o câncer de reto: avaliação dos fatores prognósticos de sobrevida em 27 doentes operados. Rev Bras Colo-proct. 2008;28(1):7-18.

4. Silva JH. Exenteração pélvica e tratamento do câncer do reto. Arq Gastroenterol. 2007;44(4):283.

5. Horta WA. Processo de Enfermagem. São Paulo: EPU;1979.

6. McEwen M. Desenvolvimento da Teoria: estruturação dos relacionamentos conceituais na enfermagem. In: McEwen M, Wills EM. Bases teóricas para a enfermagem. $2^{a}$ edição, Porto Alegre: Artmed; 2009.

7. Vitor AF, Lopes MVO, Araújo TL. Teoria do déficit de autocuidado: análise da sua importância e aplicabilidade na prática de enfermagem. Rev Esc. Anna Nery. 2010;14(3): 611-616.

8. Bulechek GM, Butcher HK, Dochterman JM. Classificação das intervenções de enfermagem (NIC), Rio de janeiro: Elsevier, 2010.
9. Moorhead S, Johnson M, Maas M. Classificação dos resultados de enfermagem (NOC), Rio de janeiro: Elsevier, 2010.

10. Lino AlA. Diagnósticos e Intervenções de Enfermagem no atendimento de indivíduos com estomas gastrointestinais: aplicando o processo de enfermagem [dissertação]. Brasília: Departamento de Enfermagem, Universidade de Brasília; 2014.

11. Carvalho VMJ, Cardoso JRS. Cuidados com dermatites periestomais. In: Malagutti W, Kakihara CT. Curativos, Estomia e Dermatologia: uma abordagem multiprofissional. São Paulo: Martinari; 2011.

12. Mauricio VC, Souza NVDO, Lisboa MTL. Determinantes biopsicossociais do processo de inclusão laboral da pessoa estomizada. Rev Bras Enferm. 2014;67(3):415-21.

13. Silva NG, Gama FA, Dutra RA. Percepção de pacientes com estomia sobre mitos e medos relacionados a esta condição. Rev Estima. 2008;6(1):22-26.

14. Garcia JBS. A dor aguda:um problema desafiador. Rev Dor. 2013;13(3):163.

15. Simões ASL. A dor irruptiva na doença oncológica avançada. Rev Dor. 2011;12(2):166-71.

\section{ERRATA}

No artigo "Exenteração Pélvica: Relato de Caso", com número de DOI: 10.5327/Z1806-3144201700010008, publicado no periódico Revista Estima, 2017;15(1):50-6, na página 50:

\section{ONDE SE LIA:}

Cristine Aves Costa de Jesus²

LEIA-SE:

Cristine Alves Costa de Jesus² 